\title{
Exposure to acetylcholinesterase-inhibiting pesticides and chronic renal failure
}

\section{Introduction}

Recent reports show an increase in the incidence of end-stage renal disease of unknown aetiology from the North-Central Province of Sri Lanka [1]. As the majority of the patients are from rural farming communities, pesticide exposure is suspected as a cause, although it is not a proven aetiological factor for chronic renal failure (CRF). Acetylcholinesterase (AChE)-inhibiting insecticides are the most widely used pesticides in Sri Lanka. Although nephrotoxicity is not recognised as a clinical feature of anticholinesterase poisoning, there are reports of acute renal failure provoked by acute tubular necrosis [2] and rabdomyolysis [3] following high dose OP exposure. This preliminary study was conducted to determine if there is an association between CRF of unknown aetiology and organophosphate (OP) exposure.

\section{Methodology}

Twenty three farmers with CRF attending the renal clinic at the Teaching Hospital, Anuradhapura (exposedCRF patients), and 18 patients from non-farming areas, attending the renal clinic of the University Medical Unit at the National Hospital, Sri Lanka (unexposed-CRF patients) were recruited for the study. Fifty healthy marine fishermen from Moratuwa, unexposed to pesticides, recruited for another study were used as an unexposed control group.

A questionnaire was administered to all subjects. Exposure to AChE-inhibiting pesticides was estimated by measuring haemoglobin-corrected red cell AChE levels (U/g) using a WHO-recommended cholinesterase test system (Test-mate, Ohio). Similar data obtained from 239 farmers from the Uda Walawe irrigation scheme recruited for a parallel study on health effects of OP exposure were used in the analysis as exposed subjects without CRF (exposed-non-CRF group). The Ethical Review Committees of the Faculty of Medical Sciences, University of Sri Jayewardenepura, and the Teaching Hospital, Anuradhapura approved the study protocol.

\section{Results}

The exposed-CRF patients were significantly older than the other subjects $(p=0.05)$. The controls were significantly younger than the CRF patients $(p=0.05$ for both exposed and unexposed patients; Table 1).

The mean number of years of cultivation among the exposed-CRF patients and exposed-non-CRF farmers was 19.27 and 15.5 years, respectively.

Haemoglobin corrected red cell AChE levels among
Table 1. Selected characteristics and red cell acetylcholinesterase (AChE) levels of subjects with and without chronic renal failure (CRF)

\begin{tabular}{|c|c|c|c|c|}
\hline \multirow[t]{3}{*}{ Characteristic } & \multicolumn{4}{|c|}{ Mean $( \pm S D)$ values in organophosphate } \\
\hline & \multicolumn{2}{|c|}{ Exposed } & \multicolumn{2}{|c|}{ Unexposed } \\
\hline & $\begin{array}{l}C R F \\
\text { patients } \\
(n=23)\end{array}$ & $\begin{array}{l}\text { Non-CRF } \\
\text { farmers } \\
(n=239)\end{array}$ & $\begin{array}{l}\text { CRF } \\
\text { patients ( } \\
(n=18)\end{array}$ & $\begin{array}{l}\text { Controls } \\
n=50)\end{array}$ \\
\hline Age (years) & $59.4^{\mathrm{a}}$ & $45.0^{\mathrm{b}, \mathrm{c}}$ & $50.1^{\mathrm{b}}$ & $39.5^{\mathrm{c}}$ \\
\hline Height $(\mathrm{cm})$ & $157.7^{\mathrm{a}, \mathrm{b}}$ & $161.7^{\mathrm{a}}$ & $155.8^{\mathrm{b}}$ & $160.8^{\mathrm{a}}$ \\
\hline Weight (kg) & $48.4^{\mathrm{a}}$ & $51.2^{\mathrm{a}}$ & $50.2^{\mathrm{a}}$ & $59.6^{\mathrm{b}}$ \\
\hline Body mass index & $19.3^{\mathrm{a}}$ & $19.5^{\mathrm{a}}$ & $20.7^{\mathrm{a}}$ & $22.9^{\mathrm{b}}$ \\
\hline $\mathrm{AChE}(\mathrm{U} / \mathrm{g})$ & $18.6^{\mathrm{a}}$ & $29.1^{\mathrm{b}}$ & $26.6^{c}$ & $32.6^{\mathrm{d}}$ \\
\hline
\end{tabular}

a,b,c,d Values having the same superscript are not significantly different at $\mathrm{p}=0.05$

farmers occupationally exposed to pesticides were significantly lower than in the unexposed controls ( $\mathrm{p}$ $<0.01$ ) (Table 1). Red cell AChE levels were lower in CRF patients (exposed and unexposed groups), as compared to the exposed non-CRF farmers and the controls $(\mathrm{p}<0.05)$. Among the patients with $\mathrm{CRF}$, red cell AChE levels were lower in the exposed group as compared to the unexposed group $(\mathrm{p}<0.05)$. The ratio of $\mathrm{AChE}$ in the exposed-non-CRF farmers to the exposed-CRF patients was 1:0.64 and, in the controls to the unexposedCRF patients was 1:0.82.

\section{Discussion}

In this study, CRF patients (both exposed and unexposed) had lower levels of red cell $\mathrm{AChE}$ as compared to non-CRF exposed farmers and controls. AChE levels were significantly lower in exposed-CRF patients as compared to unexposed-CRF patients $(\mathrm{p}<0.05)$ suggesting an association between farming and $\mathrm{AChE}$ levels. Non-CRF exposed farmers having long term lowlevel exposure to OP had significantly lower levels of AChE as compared to the controls. The finding that the AChE level in the exposed-CRF patients was significantly lower than that in unexposed-CRF patients and exposed non-CRF farmers, suggests a possible association between CRF of unknown aetiology, AChE levels and long term low-level exposure to OPs. However, whether it was indeed OP exposure that caused renal disease cannot be concluded from the results, especially because AChE levels of exposed non-CRF farmers were historical data obtained from farmers living in another part of the country.

There have been reports of OP induced nephrotoxicity. Malathion induced immune complex nephropathy has been reported previously [4]. Wedin [5] 
suggests that acetylcholine and AChE themselves can significantly alter renal function as a result of alterations in neural, humoral and metabolic activity in addition to acetylcholinesterase possibly having a direct nephrotoxic effect on renal tubules. In this study, none of the subjects reported a single episode of high dose OP poisoning.

Red cell AChE is reported to be higher in CRF patients undergoing haemodialysis and receiving recombinant erythropoietin [6]. In this study, none of the CRF patients were haemodialysed or were administered recombinant erythropoietin which probably explains the lower levels of AChE.

Although most CRF-farmers were currently not involved in pesticide spray activities, the likelihood of occupational or environmental exposure to $\mathrm{OP}$ in the Anuradhapura district is high.

The increase in pesticide use, especially OPs, since the late 1970s in Anuradhapura District has raised an important epidemiological question whether long term low-level exposure to OPs results in CRF. An extensive study is planned to assess the significance of our preliminary findings.

\section{Acknowledgements}

The authors thank Professor Rezvi Sheriff for permitting recruitment of patients from the renal clinic of the University Medical Unit, National Hospital of Sri Lanka, Colombo.

\section{References}

1. Lanerolle RD, Kudalugoda Arachchi J, Nanayakkara S, Sheriffdeen AH, Sheriff R. Demographic characteristics of end stage renal disease in Sri Lanka. Journal of the Ceylon College of Physicians 2000; 33: 124-6.

2. Betrosian A, Balla M, Kafiri G, Kofinas G, Makri R, et al. Multiple systems organ failure from organophosphate poisoning. Journal of Toxicology and Clinical Toxicology 1995; 33: 257-60.

3. Gokel Y. Subarachnoid hemorrhage and rabdomyolysis induced acute renal failure complicating organophosphate intoxication. Renal Failure 2002; 24: 867-71.

4. Albright RK. Renal involvement in organophosphate poisoning. Journal of the American Medical Association 1984; 252: 1408.

5. Wedin GP. Nephrotoxicity of anticholinesterases. In: Ballantyne B, Marrs TC, eds. Clinical and Experimental Toxicology of Organophosphates and Carbamates. Oxford: Butterworth-Heinemann, 1992: 195-202.

6. Prall YG, Gambir KK, Cruz IA, Blassingale J, Ampy FR. Acetylcholinesterase activity in chronic renal failure. Life Sciences 2000; 66: 835-45.

RJ Peiris-John, Department of Physiology, JKP Wanigasuriya, Department of Medicine, Faculty of Medical Sciences, University of Sri Jayewardenepura, AR Wickremasinghe, Department of Community and Family Medicine, Faculty of Medicine, University of Kelaniya; WP Dissanayake and A Hittarage, Teaching Hospital, Anuradhapura, Sri Lanka. Correspondence: RJP-J, e-mail: <roshi@visual.Ik>. (Competing interests: none declared). 